\title{
Organic Synthesis Facility
}

National Cancer Institute

\section{Source}

National Cancer Institute. Organic Synthesis Facility. NCI Thesaurus. Code C16037.

A core facility for the preparation and synthesis of org anic molecules. 